\title{
Measurement of the thermal conductivity of liquids by hot wire method: comparison between transient and stationary approaches
}

\author{
Jean-Pierre Monchau ${ }^{1 *}$, Nicolas Lalanne ${ }^{1}$, Laurent Ibos ${ }^{2^{*}}$ \\ ${ }^{1}$ THEMACS Engineering, 23 rue Alfred Nobel, 77420 Champs-sur-Marne, France \\ ${ }^{2}$ CERTES / OSU Efluve, Université Paris-Est Creteil, 61 avenue du Général de Gaulle, 94010 Créteil \\ Cedex, France \\ * Corresponding author: monchau@themacs.fr
}

\begin{abstract}
This work concerns the characterization of the thermal conductivity of liquids by hot wire method. A measuring cell has been specially developed for this study. The method of analysing the transient response makes it possible to obtain values of conductivity close to those expected (deviations $<4 \%$ ) for two reference fluids and measurement uncertainties in the order of $5 \%$. The use of the so-called steady state method (ASTM D 2717) leads to relative deviations and uncertainties of the measurements about 3 times higher.
\end{abstract}

\section{Nomenclature}

T temperature, ${ }^{\circ} \mathrm{C}$

I electrical current, $\mathrm{mA}$

$\mathrm{U}$ measured voltage, $\mathrm{mV}$

R electrical resistance, $\Omega$

L length of the platinum wire, $\mathrm{mm}$

$\mathrm{r}$ radius, $\mathrm{mm}$

t time $s$

A calibration constant, $\mathrm{m}^{-1}$

B calibration constant, $\mathrm{K} . \mathrm{W}^{-1}$
Greek symbols

$\lambda$ Thermal conductivity, $\mathrm{Wm}^{-1} \cdot \mathrm{K}^{-1}$

$\alpha$ slope, $\mathrm{K}$

$\beta$ resistance variation, $\%$. ${ }^{\circ} \mathrm{C}^{-1}$

$\Delta$ variation

Indices and exhibitors

f Heating wire

$\mathrm{v}$ glass

i Inner side of the glass tube

o Outer face of the glass tube

\section{Introduction}

One of the methods commonly used for measuring the thermal conductivity of liquids is the hot wire method [1,2]. This method is based on the heating by an electric current a very thin wire immersed in the liquid to be characterized. The variation of the temperature of the wire depends on the exchanges with the liquid, and therefore on the thermal conductivity of the liquid and the contact resistance. The electrical power supplied to the wire must be constant and low enough to maintain an elevation of temperature of a few degrees maximum, which makes it possible to limit convection exchanges in the liquid. The 
temperature of the wire can be measured either by a thermocouple welded to the wire, or by measuring the electrical resistance of the wire during its warming up. The conductivity of the liquid can be determined by two methods: transient or steady-state regim. A measuring cell made of glass with a $50 \mu \mathrm{m}$ diameter platinum wire was processed in this work. The measurement of the temperature of the wire is carried out by a measure of its electrical resistance over time, by a 4-wire method which makes it possible to minimize the influence of the electrical resistance of contacts. The device allows a characterization in transient or steady states. We therefore propose in this paper a comparison of these two approaches on the basis of the study of different liquids. A comparative study of measurement uncertainties is also presented. This article is however limited to a discussion about the metrological aspects of these two methods of measurement.

\section{Experimental cell developed}

A measuring cell has been specially developed for this study. The device is freely inspired from ASTM D2717 [3]. It consists of a platinum wire stretched into the liquid to be studied. The thread must be exactly centered so that it can be modeled in terms of heat exchanges for static measurements. The photographs in Figure 1 show the measuring device. The platinum wire was installed in a U-shaped tube (of inner and outer diameters of 6 and $9 \mathrm{~mm}$ respectively), made of glass, in order to facilitate its use (filling, holding on support ...). Upon measurement, the U-tube is placed in a tank filled with water whose temperature is constantly controlled.

Figure 2 shows a synoptic of the experimental device. Centering cylinders A (PTFE) have been milled exactly to the internal dimension of the glass tube $\mathbf{D}$ and were drilled in their center at the diameter of $0.1 \mathrm{~mm}$. The centering accuracy is therefore of $25 \mu \mathrm{m}$. This represents an error of less than $1 \%$ on the inner radius of the glass tube. Platinum wire $\mathbf{C}$ is connected like a 4-wire electrical resistor in order to obtain an accurate resistance value of the portion studied. Moreover, this wiring makes it possible to limit the effects of longitudinal conduction temperature. Liquid $\mathbf{B}$ fills the glass tube. The test body is located between the two brazes that define the length $\mathbf{L}$ of measurement.

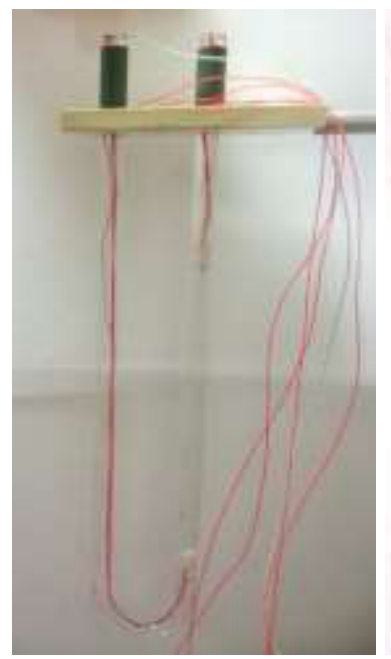

Figure 1 : left: the entire device in a U-shaped tube for easy filling; Right: detail of brazing of the two platinum wires delimiting the measuring zone

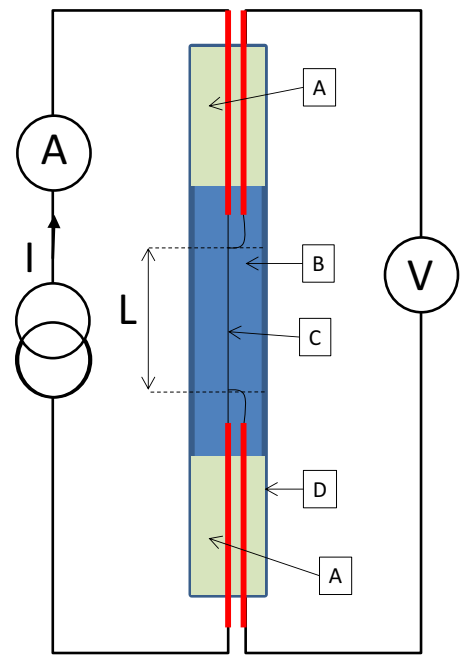

Figure 2: Block diagram of the measuring cell (see text for details) 


\section{Measurement principles and instrumentation of the device}

In both cases studied (transient or stationary measurements), the heating wire is powered by an electric current of constant intensity $I$. The voltage $U$ at the terminals of the wire is measured over time to determine changes in its electrical resistance $R$. In transient mode, a short sampling period and a short measurement duration are chosen. For stationary measurements, the measurement duration is extended until reaching a constant temperature of the wire. The processing of the experimental data differs for these two types of measurement.

\subsection{Analysis of data by transient method}

In the case of a transient measurement, a heat transfer is assumed in the fluid in cylindrical geometry in a semi-infinite medium. The measure must therefore be carried out over a short period so as not to suffer the effects of the outer glass tube. We also consider that the initial temperature of the wire $T(0)$ and the electrical power $P$ are constant. Solving the heat equation for these conditions provides an analytical expression for the time course $T(t)$ of the temperature of the heating wire. If a very thin heating wire is used, its inertia has no manifestation measurable on the temperature curve for very short times (here for $t<2 \mathrm{~s}$ ). So if we take the so-called "quasi-stationary" hypothesis, we obtain a simplified expression of the temperature variation of the wire $[1,2]$ :

$$
T(t)-T(0)=\alpha \times \ln (t)+C
$$

Where $\alpha$ is the slope of the linear portion of the temperature plot with respect to the logarithm of the time:

$$
\alpha=\frac{P}{4 \pi \lambda L} \Leftrightarrow \lambda=\frac{P}{4 \pi \alpha L}
$$

The value of $\alpha$ is obtained by linear regression (least squares) by considering portions of the curve $\Delta T=\mathrm{f}(\ln t)$ until "unsigned" residuals are obtained. Equation (2) thus allows the calculation of the thermal conductivity of the liquid $\lambda$ from the value of $\alpha$ experimentally determined and the knowledge of the length of the wire $L$ and of the power electric power $P$. The constant parameter $C$ in equation (1) further depends on the contact resistance between the wire and the liquid, the radius of the wire and the thermal diffusivity of the liquid. The values of this parameter will not be analyzed in this work. A similar method has been used by the NIST for thermal conductivity measurements of liquids [4]. This method uses two wires of different lengths placed on the two branches of a Wheatstone bridge to eliminate edge effects.

\subsection{Analysis of stationary data}

The data processing used in this case is described in ASTM D2717 [3]. The determination of the thermal conductivity of the liquid $\lambda$ is based on the measurement of total change in 
temperature $\Delta T$ of the heating wire between the initial state and the return to steady stationary and on the knowledge of two constants $A$ and $B$ characteristics of the measurement cell [3]:

$$
\lambda=\frac{A}{\Delta T / P-B}
$$

The constants $A$ and $B$ are defined from the dimensions of the measuring cell and the thermal conductivity of the glass constituting the U-tube [3]:

$$
A=\frac{\ln \left(r_{i} / r_{f}\right)}{2 \pi L} ; B=\frac{\ln \left(r_{o} / r_{i}\right)}{2 \pi \lambda_{v} L}
$$

The ASTM standard alternatively proposes making a determination of the constants $A$ and $B$ by a measurement on one or more reference liquids, the most important influence parameter being the parameter $A$.

\subsection{Instrumentation of the measuring cell}

A current source (Keithley 2400) was used to apply a current transient method and a permanent current for the static method. A voltmeter (Keithley 2182) makes it possible to measure the voltage at the terminals of the portion of the wire used to carry out the measurement. This voltmeter has a resolution of $10 \mathrm{nV}$ and therefore allows to measure small variations of the voltage at the terminals of the wire. Both devices are controlled by a LabView application via a GIPB bus link. The measurement is carried out in two steps.

First, a low current $(<1 \mathrm{~mA})$ is applied for 8 seconds to obtain the initial value of the electrical resistance of the wire, and thus of its temperature. The current value has been selected sufficiently low so as not to generate self-heating detectable during this first step and sufficiently high to allow a measurement of the voltage with a satisfactory signal-tonoise ratio. Knowledge of the initial resistance of the wire is required for the static method described in the ASTM standard.

In a second step, a higher constant current is applied in order to generate a heating of the platinum wire. The measuring step (from $62.5 \mathrm{~ms}$ to $833 \mathrm{~ms}$ ) and the total duration (from $64 \mathrm{~s}$ to $833 \mathrm{~s}$ ) depend on the chosen method (transient or stationary). The intensity of the current can be chosen between $10 \mathrm{~mA}$ and $1 \mathrm{~A}$. The value must be adapted to the conductivity of the liquid in order to limit the rise in temperature to a few degrees $\mathrm{C}$.

The temperature of the hot wire is calculated from the resistance measurements using standard values for the variation of the platinum resistivity of the temperature. For this material, it is possible to consider, in a reduced temperature range around the ambient temperature, a coefficient of variation of the electrical resistance $\beta=0.3862 \%$. ${ }^{\circ} \mathrm{C}^{-1}$. The use of this coefficient makes it possible to transform the measured electrical resistance relative variations of the measured electrical resistance $\Delta R / R$ into a temperature variation $\Delta T$. The temperature of the liquid in the U-tube and the temperature of the water in the vessel containing the U-tube are controlled during the experiments using thermocouples and a Fluke $^{\mathrm{TM}}$ Digital Recorder. It is necessary to ensure that the temperature of the water in the vessel remains constant for the duration of an experiment. This is particularly important in the case of steady state measurements. The maximum variation of the temperature tolerated in this study was chosen to be within $\pm 0.2^{\circ} \mathrm{C}$. 


\section{Results}

\subsection{Liquids studied}

Two liquids were used in this study: demineralized water and dimethylphthalate (DMP). The thermal conductivities of these two liquids are tabulated as a function of temperature in ASTM D2717 [3]. This will allow us to compare the results of the measurements to reference values.

\subsection{Thermal conductivity measurements}

\subsubsection{Results obtained with transient method}

Figures 3 and 4 show the temperature variations of the wire obtained for both liquids for two filament feed streams as well as regressions carried out on the linear portions of the curves. In both cases, the increase in temperature on the linear portion is about $1.2^{\circ} \mathrm{C}$.

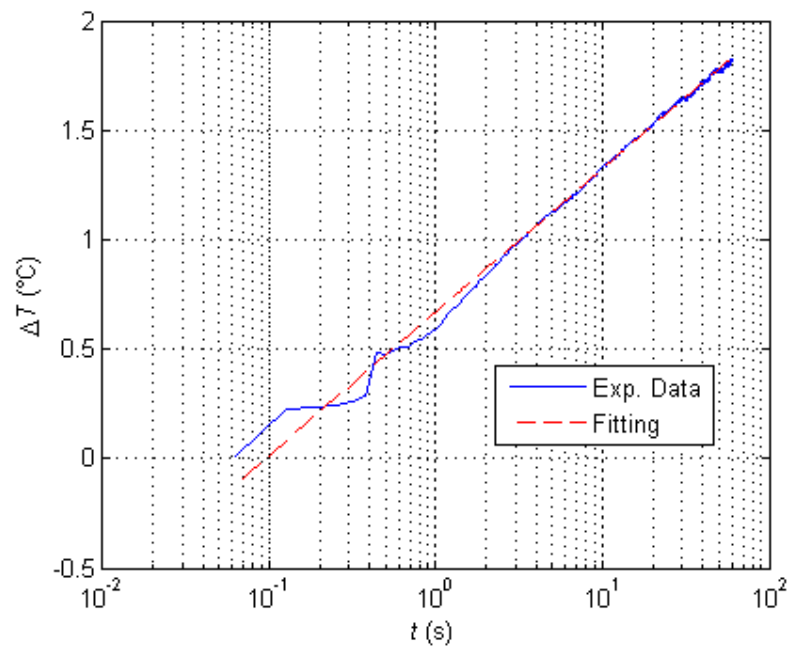

Figure 3: Temperature of the hot wire measured for water with $\mathrm{I}=200 \mathrm{~mA}$ 


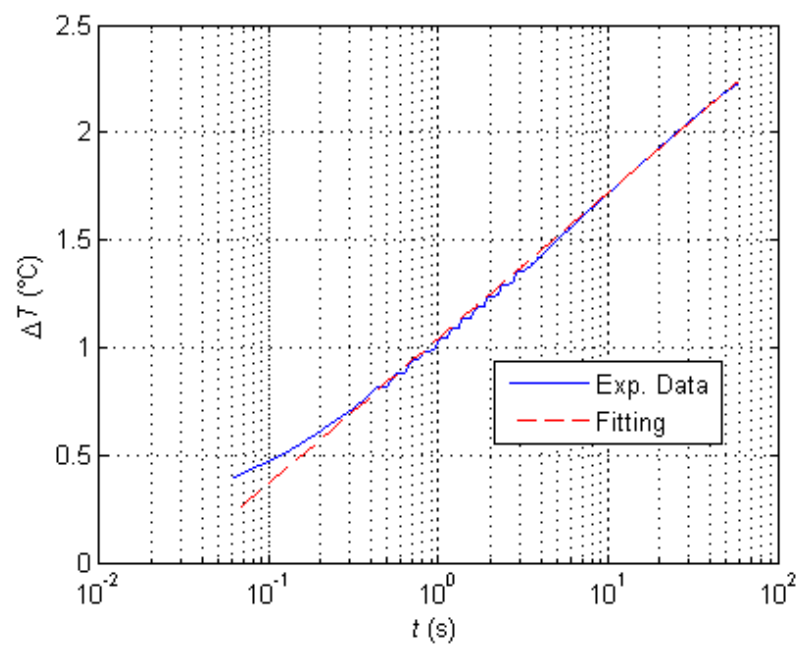

Figure 4: Temperature of the hot wire measured for the DMP with $I=100 \mathrm{~mA}$

Table 1: Thermal Conductivity of the water and the DMP for transient method with different wire currents; Comparison with literature data.

\begin{tabular}{clllll}
\hline & $I$ & $\lambda_{\text {measured }}$ & $\lambda_{\text {reference }}[3]$ & $\Delta \lambda / \lambda$ \\
\cline { 2 - 5 } & $\mathrm{mA}$ & $\mathrm{W}^{-1} \mathrm{~m}^{-1} \cdot \mathrm{K}^{-1}$ & $\mathrm{~W} \cdot \mathrm{m}^{-1} \cdot \mathrm{K}^{-1}$ & $\%$ \\
\hline \multirow{2}{*}{ Water } & 100 & 0.593 & 0.610 & -2.8 \\
\cline { 2 - 5 } & 200 & 0.613 & 0.613 & 0.0 \\
\cline { 2 - 5 } & 300 & 0.639 & 0.616 & 3.7 \\
\hline \multirow{2}{*}{$\begin{array}{c}\text { Dimethyl } \\
\text { Phtalate }\end{array}$} & 50 & 0.145 & 0.148 & -1.7 \\
\cline { 2 - 5 } & 100 & 0.147 & 0.148 & -0.6 \\
\cline { 2 - 5 } & 150 & 0.143 & 0.147 & -3.2 \\
\hline
\end{tabular}

The thermal conductivity values obtained for the two liquids and different filament feed currents are shown in Table 1. Currents twice weaker have been selected for DMP in order to maintain temperature variations similar to those obtained for water, the expected thermal conductivity of the DMP being about 4 times lower than that the one of water. It can be seen that there is a dispersion of values without a tendency to diminish or increase the results. The method seems therefore unbiased but a notable dispersion exists (up to 3.7\%). Note that the ASTM standard announces a scatter of results up to $10 \%$.

\subsubsection{Results obtained in steady state}

Steady-state measurements were performed for both liquids, considering the value of the electric current allowing to obtain the transient measurement closest to the reference value. The measurement is continued until a stable value of the resistance of the filament is reached. The final value of the resistance is obtained by taking the long time asymptotic value. For this experiment, it is also necessary to use a power supplied to the wire as stable as possible. Figures 5 and 6 show respectively the variation of resistance and of power measured over time for water with a supply current of $200 \mathrm{~mA}$. For $T>400 \mathrm{~s}$, we obtain 
variations of the wire resistance less than $0.3 \%$ and of the power less than $0.02 \%$, which makes it possible to consider that a steady state is reached. The thermal conductivity values obtained from the steady state measurements are presented in Table 2 for both liquids considered. The total temperature variation $\Delta T$ obtained is also reported in the table. Compared to results obtained by a transient analysis, a larger gap between measured and expected values is observed. This discrepancy does not seem to be explained by a systematic error.

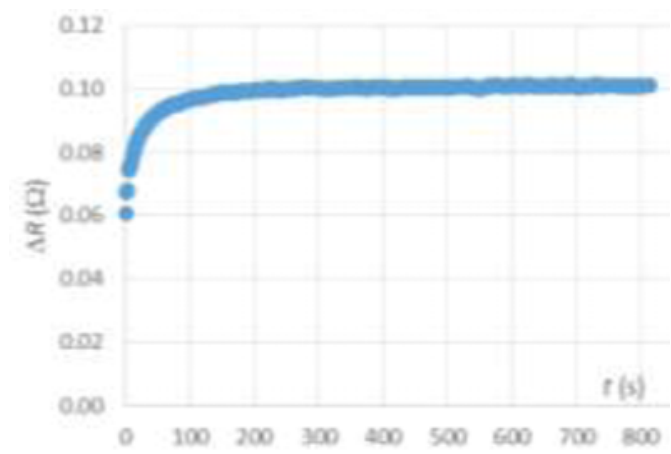

Figure 5: variation in resistance of the wire measured for stationary water for $I=200$ $\mathrm{mA}$

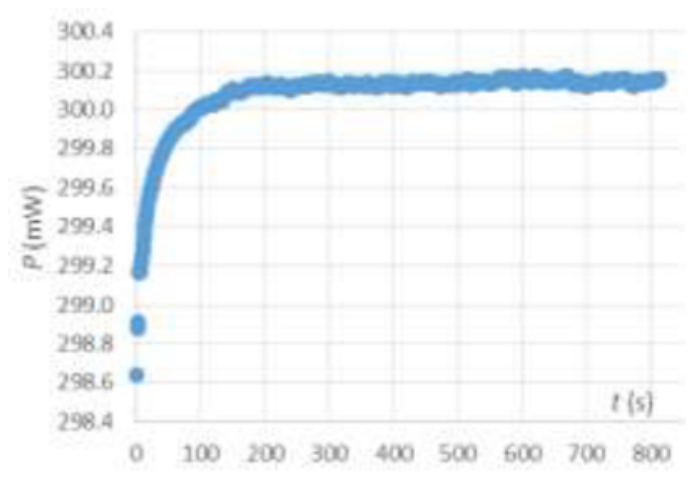

Figure 6: Variation in power of the hot wire measured for stationary water for $I=200$ $\mathrm{mA}$ 
Table 2: Measurement of thermal conductivity of water and dimethyl phthalate with the stationary method; Comparison with data in the literature

\begin{tabular}{clllcl}
\hline & $I$ & $\Delta \mathrm{T}$ & $\lambda_{\text {measured }}$ & $\lambda_{\text {reference }}[3]$ & $\Delta \lambda / \lambda$ \\
\cline { 2 - 6 } & $\mathrm{mA}$ & ${ }^{\circ} \mathrm{C}$ & $\mathrm{W} \cdot \mathrm{m}^{-1} \cdot \mathrm{K}^{-1}$ & $\mathrm{~W} \cdot \mathrm{m}^{-1} \cdot \mathrm{K}^{-1}$ & $\%$ \\
\hline Water & 200 & 3.58 & 0.555 & 0.613 & -9.5 \\
\hline Diméthyl Phtalate & 100 & 2.80 & 0.170 & 0.148 & 15 \\
\hline
\end{tabular}

\subsection{Analysis and evaluation of measurement uncertainties}

The determination of the conductivity by stationary or transient methods involves several influencing parameters which can be classified into four categories:

- The dimensions of the measuring cell: the length of the heating wire, the radius of the wire and glass tube (stationary measurements only). These quantities were measured and uncertainty calculated.

- The properties of the materials used: temperature coefficient of the platinum wire and thermal conductivity of glass (stationary measurements only); these parameters were not measured, the values used were taken from the literature and the uncertainties fixed to $3 \%$ for $\beta$ and to $20 \%$ for $\lambda_{\mathrm{v}}$.

- The measured quantities: current applied to the heating wire and voltage measured at the wire terminals. The uncertainties of these quantities were established on the basis of the technical datasheets of the current source and voltmeter on one hand and a calculation of the standard deviation on measured values (in the case of steady state tests) on the other hand.

- Intermediate quantities calculated from measurements and / or fixed quantities. Using a conventional uncertainty propagation technique as defined in the reference [5], we obtain the uncertainties presented in Table 3 for some situations (a transient measurement and a stationary measurement for both liquids). The uncertainty of transient measurement is identical for the two liquids used. The uncertainty obtained by the stationary method is much higher and is depending on the liquid used. Moreover, these uncertainties are greater than the relative observed variability between the measured values and the expected values. These gaps are therefore probably related to uncertain parameters used for the calculation of thermal conductivity. 
Table 3: Measurement uncertainties on the thermal conductivity of the liquid

\begin{tabular}{clll}
\hline & $I$ & $\begin{array}{l}\text { Measurement } \\
\text { method }\end{array}$ & $\begin{array}{l}\text { Uncertainty } \\
\text { on } \lambda\end{array}$ \\
\cline { 2 - 4 } & $\mathrm{mA}$ & - & $\%$ \\
\hline \multirow{2}{*}{ Water } & 200 & transient & 5.2 \\
\cline { 2 - 4 } & 200 & stationary & 13 \\
\hline Diméthyl Phtalate & 100 & transient & 5.2 \\
& 100 & stationnaire & 17 \\
\hline
\end{tabular}

An assessment of the respective contributions of the influencing parameters on the final uncertainty estimated for the thermal conductivity is presented for the case of the measurements in transient regime in figure 7. A similar balance is shown in figure 8 for a measurement in stationary regime. The data considered for each influencing parameter is the product of the standard uncertainty by the sensitivity coefficient (in absolute value). The values are then standardized to 100 for the most influencing parameter contribution. As an indication, Table 4 provides the values of the various physical parameters and their uncertainties (the case of characterization of dimethyl phthalate is considered only). For the transient method, the reduction of uncertainties requires a calibration of the variation of the electrical resistance of the wire as a function of the temperature (parameter $\beta$ ) and by an improvement of the method used for the estimation of the slope $\alpha$. Uncertainty over the length of the wire is more difficult to reduce. For the static method, the uncertainty is mainly due to the estimated absolute resistances of initial and final resistances, leading to significant relative uncertainties on $\Delta R$ and $\Delta T$. This problem is intrinsic to this method.

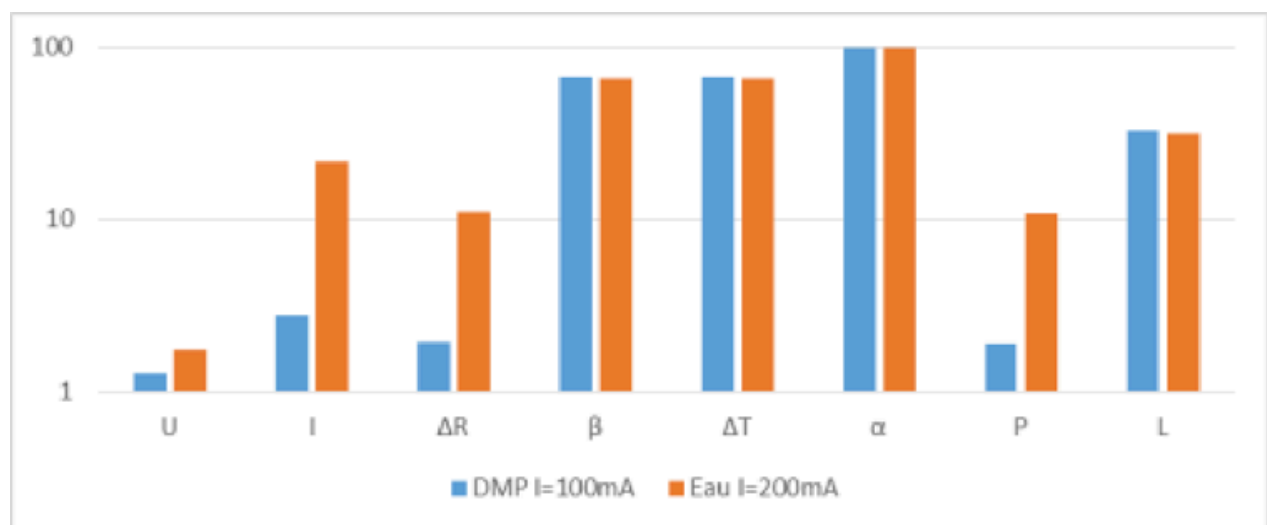

Figure 7: Relative contributions to measurement uncertainty on the thermal conductivity in the case of two transient measurements 


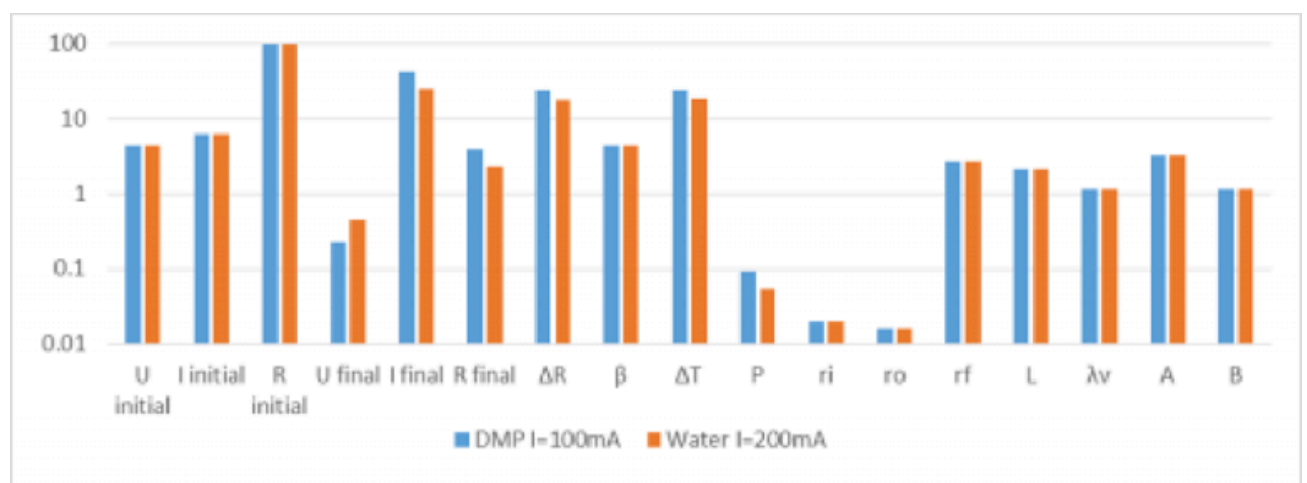

Figure 8: Relative contributions to the measurement uncertainty on the thermal conductivity in the case of two steady-state measurements

Table 4: Values of physical parameters and their uncertainties (see text for more details)

\begin{tabular}{llll}
\hline Cell parameters & Stationary measurement & & Transitional measure \\
\hline$L=137.5 \pm 2.0 \mathrm{~mm}$ & $U_{\text {init }}=7.3562 \pm 0.0005 \mathrm{mV}$ & $A=6.34 \pm 0.15 \mathrm{~m}^{-1}$ & $U=743.77 \pm 0.42 \mathrm{mV}$ \\
\hline$r_{0}=4.50 \pm 0.01 \mathrm{~mm}$ & $I_{\text {init }}=1000.00 \pm 0.95 \mu \mathrm{A}$ & $B=0.469 \pm 0.094 \mathrm{~K} . \mathrm{W}^{-1}$ & $I=99.997 \pm 0.061 \mathrm{~mA}$ \\
\hline$r_{\mathrm{i}}=3.00 \pm 0.01 \mathrm{~mm}$ & $R_{\text {init }}=7.356 \pm 0.011 \square$ & $P=74.291 \pm 0.045 \mathrm{~mW}$ & $P=74.375 \pm 0.062 \mathrm{~mW}$ \\
\hline$r_{\mathrm{f}}=25.0 \pm 0.25 \mu \mathrm{m}$ & $U_{\text {final }}=742.93 \pm 0.03 \mathrm{mV}$ & $\Delta R=73 \pm 12 \mathrm{~m} \Delta$ & $\Delta R=53.046 \pm 0.045 \mathrm{~m} \Omega$ \\
\hline$\lambda_{\mathrm{v}}=1.0 \pm 0.2 \mathrm{~W} \cdot \mathrm{m}^{-1} . \mathrm{K}^{-1}$ & $I_{\text {final }}=100.00 \pm 0.06 \mathrm{~mA}$ & $\Delta T=2.80 \pm 0.46^{\circ} \mathrm{C}$ & $\Delta T=2.028 \pm 0.061^{\circ} \mathrm{C}$ \\
\hline$\beta=0.386 \pm 0.012 \square .{ }^{\circ} \mathrm{C}^{-1}$ & $R_{\text {final }}=7.4295 \pm 0.0046 \Omega$ & - & $\alpha=0.293 \pm 0.013{ }^{\circ} \mathrm{C}$ \\
\hline
\end{tabular}

\section{Conclusion}

The measuring cell is simple to design and use. The gap between measurements obtained by transient method and the corresponding expected values for each liquid tested is lower than the measurement uncertainties. The number of influencing parameters is higher in the case of steady-state tests (17) than in the case of transient measurements (8), which leads to greater measurement uncertainties. In addition, this method tends to encourage convective exchanges in the liquid, which are not taken into account in the models. A more in-depth study is needed to quantify the influence of this phenomenon on measurements. The use of a transient method thus seems preferable to the use of the method of ASTM D 2717 [3]. On the other hand, other measuring approaches can also be tested (harmonic excitation, 3- $\omega$ method for example) with this device. Similarly, a comparison of measurements with other existing devices (commercial devices, miniature cells, etc.) is programmed. 


\section{References}

[1] B. Neindre, Measurement of the thermal conductivity of liquids and gases, Techniques the engineer, R2920-V2 (1996).

[2] Standard ASTM D 5930, Standard test method for thermal conductivity of plastics Transient line-source technique (January 2009).

[3] Standard ASTM D 2717, Standard test method for thermal conductivity of liquids (January 1995).

[4] HM. Roder, A transient hot-wire thermal conductivity apparatus for fluids, Journal of research

of the National Bureau of Standards, Vol. 86, No. 5, p. 457-493 (1981).

[5] GUM 1995, Evaluation of measurement data - A guide to the expression of uncertainty Measure, JCGM 100: 2008 (E), Doc. BIPM. 